\title{
Formation of Dynamic Waveguide Structures in Media with Thermal and Resonant Nonlinearity
}

\author{
D. V. Gorbach ${ }^{a}$, O. G. Romanov ${ }^{a}$, A. P. Sukhorukov ${ }^{b}$, and A. L. Tolstik ${ }^{a}$ \\ ${ }^{a}$ Belarussian State University, Minsk, 220030 Belarus \\ ${ }^{b}$ Moscow State University, Moscow, 119991 Russia \\ e-mail:gorbach@tut.by; romanov@bsu.by; apsmsu@gmail.com
}

\begin{abstract}
We investigate the formation of optical dynamic waveguide structures in media with thermal and resonant nonlinearities. An optical waveguide is formed experimentally in absorbing a solution of organic dye with negative thermo-optical coefficient under a powerful singular light beam (an optical vortex).
\end{abstract}

DOI: $10.3103 / \mathrm{S} 106287381112015 \mathrm{X}$

\section{INTRODUCTION}

Much attention is now being given to systems based on the strategy of controlling light with light [1-3]. Studying the effects of reflection, tunneling, and diffraction upon the interaction of optical beams in nonlinear media is of special interest [4-6]. The implementation of waveguide propagation of one light beam in the region of optical inhomogeneity induced by another beam is an interesting manifestation of nonlinear interaction effects of light beams. These investigations are intriguing as they expand the possibilities of optical switching and using dynamic waveguide structures in microparticle manipulation [7].

We conducted theoretical and experimental research on the processes of forming dynamic waveguide structures in media with resonant thermal nonlinearity and analyzed the possibility of controlling the space structure of signal light beam upon its propagation in a localized spatial region with the waveguide profile of a refractive index, induced by a powerful pump beam. An absorbing solution of organic dye with a negative thermo-optical coefficient $(d n / d T<0)$ was selected to form the waveguide structure upon the treatment of a powerful singular light beam.

\section{THEORETICAL MODEL}

In order to describe the formation of spatially inhomogeneous waveguide structures of the refractive index theoretically, we assume that powerful pump wave $E_{1}$ propagating in a nonlinear medium is characterized by optical frequency $\omega_{1}$ that coincides with the center of absorption line $S_{0}-S_{1}$ of the dye solution. The probe wave $E_{S}$ at frequency $\omega_{S}$ for which the medium is transparent has a collinear direction to the pump wave. In theoretical modeling, we consider that in addition to resonant nonlinearity, thermal nonlinearity plays a large role in forming the optical response for dye solutions.
The expressions for nonlinear medium susceptibility at the frequencies $\omega_{1}$ and $\omega_{S}$ take the following form under these conditions:

$$
\begin{gathered}
\chi\left(\omega_{1}\right)=\frac{n_{0} \kappa_{0}}{2 \pi}\left(\frac{\bar{\Theta}_{12}}{B_{12}}-\frac{\bar{\alpha} I}{1+\alpha I}\right), \\
\chi\left(\omega_{S}\right)=\frac{n_{0} \kappa_{0}}{2 \pi} \frac{a_{T} I}{1+\alpha I},
\end{gathered}
$$

where $\bar{\alpha}=a+i \alpha=\left(\bar{\Theta}_{12}+\bar{\Theta}_{21}\right) / v P_{21}-a_{T}, \bar{\Theta}_{k l}(\omega)=$ $\Theta_{k l}(\omega)+i B_{k l}(\omega)$. Coefficients $\Theta_{k l}(\omega)$ are associated by the Kramers-Kronig relations with Einstein coefficients for stimulated transitions $B_{k l}(\omega)$ in spectral channel $S_{0}-S_{1} ; \mathrm{v}=c / n_{0}$ is the velocity of light in the medium, $\kappa_{0}$ is the initial extinction ratio; $n_{0}$ is the reflective index; and $P_{21}$ is the total probability of spontaneous and nonradiative transitions. The value $\alpha==\left(B_{12}+B_{21}\right) / \nu P_{21}$ defines the intensity of saturation of resonant transition $\left(I_{\text {sat }}=\alpha^{-1}\right)$. The $a_{T}=2 \omega\left(1-\mu_{21}\right)(d n / d T) \tau / c C_{\rho}$ coefficient characterizes the thermal change in the reflective index of dye solution. Here, $\tau$ is the duration of interaction, $C_{\rho}$ is the heat capacity of unit volume, $d n / d T$ is the thermo-optical coefficient, and $\mu_{21}$ is the quantum yield of luminescence in the $S_{0}-S_{1}$ channel.

According to the nonlinear modulation of the coefficient of absorption and the revlective index in a powerful pump wave field, equations for complex amplitudes of light waves $E_{1}$ and $E_{S}$ can be written in the following form:

$$
\begin{gathered}
\frac{\partial E_{1}}{\partial z}+\frac{1}{2 i k_{1}} \Delta_{\perp} E_{1}=\frac{i 2 \pi \omega_{1}}{c n_{0}} \chi\left(\omega_{1}\right) E_{1} \\
\frac{\partial E_{S}}{\partial z}+\frac{1}{2 i k_{S}} \Delta_{\perp} E_{S}=\frac{i 2 \pi \omega_{S}}{c n_{0}} \chi\left(\omega_{S}\right) E_{S},
\end{gathered}
$$

where $k_{1}=\omega_{1} / \nu$ and $k_{S}=\omega_{S} / \nu ; \Delta_{\perp}=\partial^{2} / \partial x^{2}+$ $\partial^{2} / \partial y^{2}$ is a transverse Laplacian. 
(a)
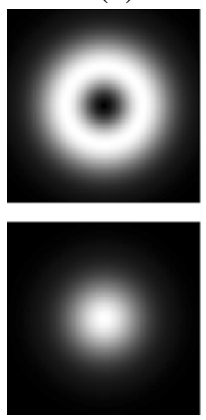

(b)

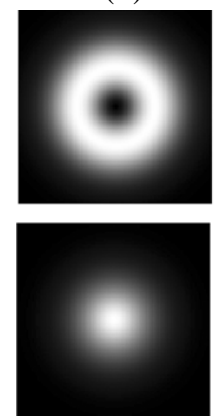

(c)

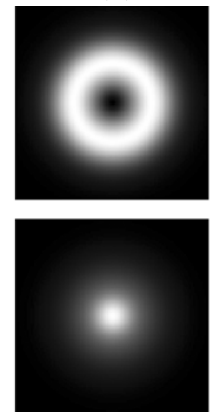

(d)

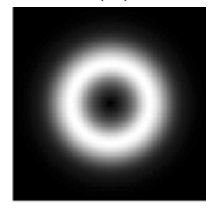

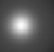

Fig. 1. Distribution of light beam intensity at output (a) and input $(b-d)$ of a nonlinear medium: $\alpha I_{01}=1$ (b), $5(\mathrm{c})$, and $30(\mathrm{~d})$.

Considering the explicit form of expressions for nonlinear susceptibility $\chi(\omega)$ in (1) and $\chi\left(\omega^{\prime}\right)$ in (2), the Eqs. (3) system of a coupled wave becomes

$$
\begin{gathered}
\frac{\partial E_{1}}{\partial \zeta}=i \Delta_{\perp}^{\prime} E_{1}+i k_{a} L_{D 1}\left(\frac{\bar{\Theta}_{12}}{B_{12}}-\frac{\bar{\alpha} I_{1}}{1+\alpha I_{1}}\right) E_{1}, \\
\frac{\partial E_{S}}{\partial \zeta}=i \Delta_{\perp}^{\prime} E_{S}+i k_{a} L_{D S}\left(\frac{a_{T} I_{1}}{1+\alpha I_{1}}\right) E_{S},
\end{gathered}
$$

where $\Delta_{\perp}^{\prime}=\partial^{2} / \partial \xi^{2}+\partial^{2} / \partial \eta^{2}$. The spatial coordinates are normalized as follows: $\xi=x / r_{0}, \eta=y / r_{0}$, and $\zeta=$ $z / 2 L_{D 1}$. Diffraction wave $L_{D 1}=2 \pi r_{0}^{2} / \lambda_{1}$ is determined by the width of the pump beam $r_{0} ; L_{D S}=2 \pi r_{0}^{2} / \lambda_{S}$; and $k_{a}$ is the coefficient of absorption of the pump radiation.

It is assumed in the numerical modeling of the Eqs. (4) system that the light pump beam on wavelength $\lambda_{1}=532 \mathrm{~nm}$, which is directed to the $z=0$ boundary of the nonlinear medium, is characterized by an intensity distribution that is tubular in cross section and contain a phase screw dislocation on the order of $m: E_{1}(z=0)=$ $E_{01}\left[r / r_{01}\right]^{|m|} \times \exp \left[-\left(r / \sqrt{2} r_{01}\right)^{2}+i m \varphi\right]$ (Fig. 1a, upper row). The signal beam on the wavelength $\lambda_{S}=0.63 \mu \mathrm{m}$ at the input of the nonlinear medium has a Gaussian intensity profile: $E_{S}(z=0)=E_{0 S} \exp \left[-\left(r / \sqrt{2} r_{0 S}\right)^{2}\right]$ (Fig. 1a, lower row). The half-width of the light beams was taken to be $r_{01,0 S}=200 \mu \mathrm{m}$; the peak beam intensity varied within $\alpha I_{01}=1-100$; the initial coefficient of absorption was selected on the order of $k_{a}=1 \mathrm{~cm}^{-1}$; and the spectroscopic and thermo-optical parameters of nonlinear medium corresponded to an ethanol solution of $6 \mathrm{G}$ rhodamine.

Figure $1 b-d$ presents the results from numerical calculations of the intensity distributions (white denotes the maximum values) in the pump beam (upper row) and in the signal beam (lower row) at the ouput of the nonlinear medium $z=0.1 L_{D 1}$, These distributions were obtained at different values of the

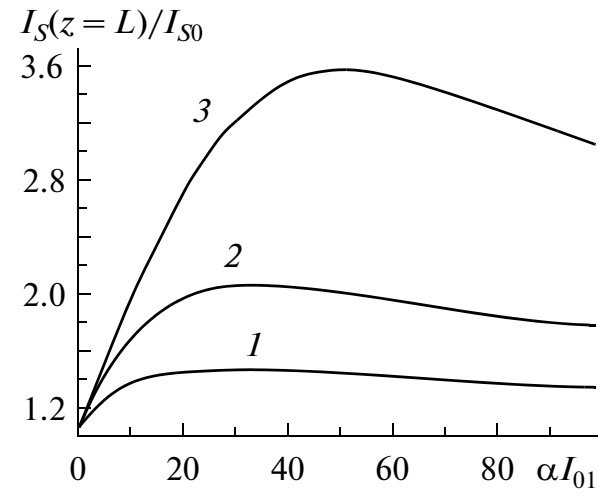

Fig. 2. Relation between the extent of signal beam focusing and pump beam intensity. (1) $k_{a}=0.5 \mathrm{~cm}^{-1}$, (2) $1 \mathrm{~cm}^{-1}$, and (3) $2 \mathrm{~cm}^{-1}$.

intensity of the pump beam at the input. Despite the presence of absorption, the singular pump beam propagating in nonlinear medium is seen to retain the circular distribution of intensity in its cross section. In this case, the spatial profile of refractive index in the defocused medium formed by the powerful pump beam is akin to the profile of the optical waveguide (the maximum $n$ value is reached along the axis of the light beam). The signal light beam captured in the region of the light-induced waveguide is localized along the transverse dimensions are defined by the pump beam (Fig. 1b-d, lower row).

Note that the reduced effectiveness of signal beam focusing (Fig. 2) caused by the effect of absorption saturation and the profile distortion of the optical waveguide is observed at values of pump intensity that appreciably exceed the saturation intensity $\left(\alpha I_{01}>\right.$ 30-50]). As this occurs, the extent of focusing (the ratio between the peak intensity of the signal beam at the output of the nonlinear medium and the peak intensity at the input, $\left.I_{S}(z=L) / I_{S 0}\right)$ depends considerably on the coefficient of absorption of nonlinear medium and can be varied within wide limits by changing the pump intensity.

\section{RESULTS AND DISCUSSION}

Figure 3 presents our experimental setup for studying the process of forming a waveguide structure in a dye solution. The continuous radiation of heliumneon laser 1 at wavelength $\lambda_{S}=632.8 \mathrm{~nm}$ was used as the signal beam. The powerful light pulse at wavelength $\lambda_{1}=532 \mathrm{~nm}$ was generated by yttrium-aluminum-garnet laser 2. The laser acted in the mode of active Q-switching with generation frequency doubling (pulse duration, $\tau=20 \mathrm{~ns}$; laser beam intensity divergence angle, 1.5 millirad; pulse repetition rate, $v=1-10 \mathrm{~Hz}$ ). Holographic transparency 3 converted the pump laser radiation to a singular light beam with topological charge $m=1$. Long-focus lens 4 was used 


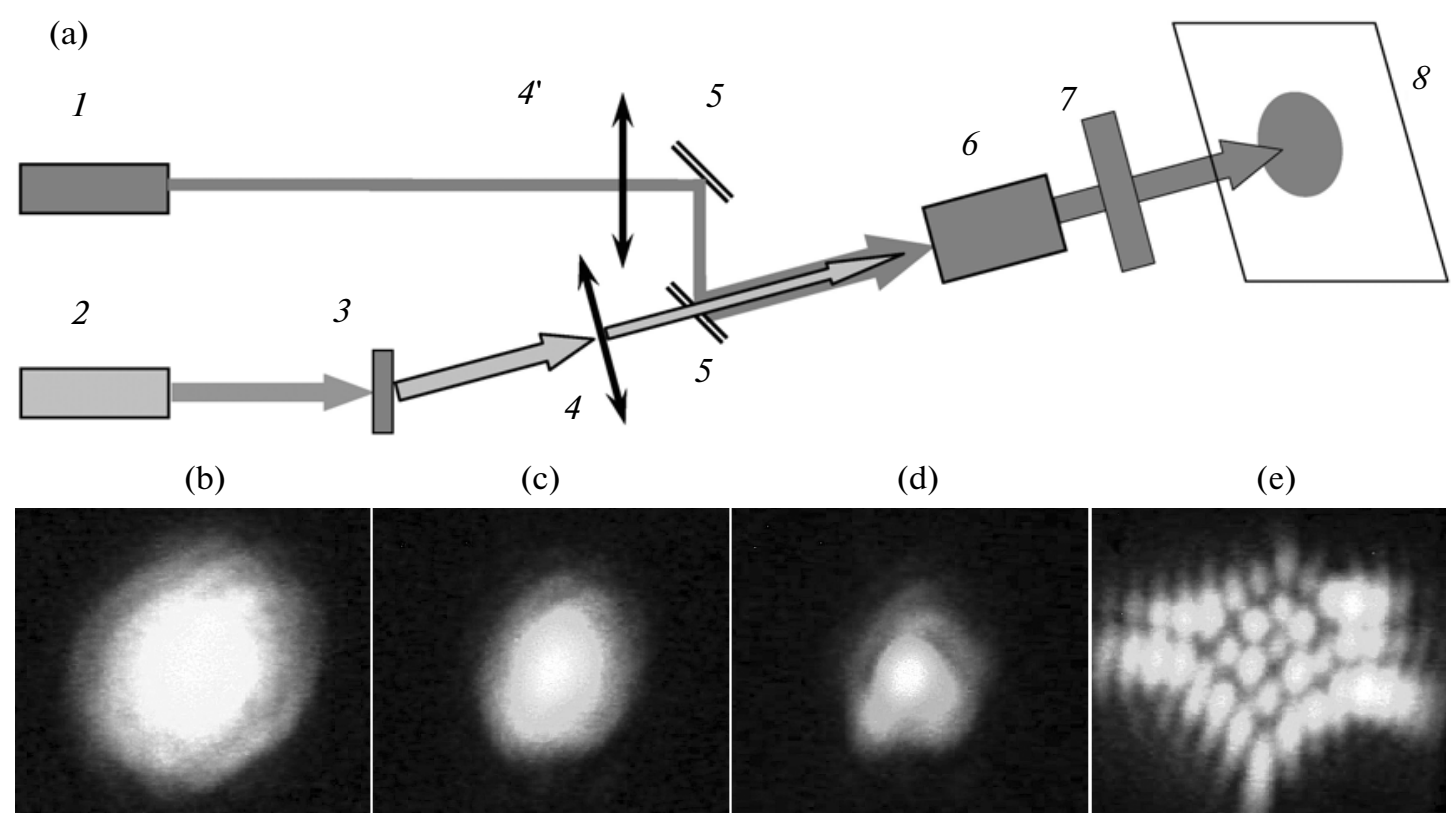

Fig. 3. Circuit of the nonlinear interaction of light beams (a) ((1) He-Ne laser; (2) YAG laser; (3) optical transparency; (4) and (4') lenses; (5) mirrors; (6) nonlinear medium; (7) light filter; (8) screen) and the form of signal beam intensity distribution at the output from the nonlinear medium (b) in the absence of a pump beam and at pump intensities (c) $I=40 \mathrm{MW} \mathrm{cm}^{-2}$ and $(\mathrm{d}, \mathrm{e}) 70 \mathrm{MW} \mathrm{cm}^{-2}$. The focuses of the signal and pump beams were spaced at $2 \mathrm{~cm}(\mathrm{~b}-\mathrm{d})$ or coincided (e).

to increase the pump power. Lens 4 ' was designed for matching the transverse dimensions of the signal and pump beams. System of moving mirrors 5 allowed us to change the angle between the interacting beams and the point of their interaction in the cell with nonlinear medium 6 . The cell length was $5 \mathrm{~cm}$. An ethanol solution of $6 \mathrm{G}$ rhodamine, which effectively absorbs radiation at the wavelength of pump laser generation, was used as the nonlinear medium, since it was practically transparent to the signal beam. The resulting interaction was recorded on screen 8 using a camera as the pump radiation transmitted through the cell was cut off by light filter 7 .

During the experiment, the outside diameter of the singular pump beam at the input to the nonlinear medium was $400 \mu \mathrm{m}$, while the inside core diameter was $200 \mu \mathrm{m}$. When propagating in the absorbing solution of organic dye with the negative thermo-optical coefficient $(d n / d T<0)$, a laser pulse with a circular transverse distribution of intensity leads to the nonuniform distribution of temperature in the volume of nonlinear medium. This results in a localized spatial region with a gradient of the refractive index that is similar to the profile of the refractive index of a typical optical waveguide. If the signal light beam is directed toward such a phase-modulated medium, the effects of full or partial reflection can be manifested in accordance with the geometry, as can the waveguide propagation.

The use of signal beam with transverse dimensions larger or equal to the dimensions of the pump beam, leads to the ejection of signal beam radiation from the region of selfinduced change in the reflective index induced by the power beam. Different modes of interaction analogous to those of incoherent Gaussian light beams from [5] are observed when we reduces the dimensions of signal beam to $200 \mu \mathrm{m}$, which corresponds to the dimensions of the core of the circular dinstribution of pump beam intensity. When both beams are directed toward the medium in alignment, the change in the transverse distribution of signal beam intensity at the output from the nonlinear medium upon increasing the power of the pump beam confirms the formation of the waveguide structure of the change in the refractive index in the medium (Fig. 2b-d).

The following geometry was applied for the effective input of signal beam radiation into the induced optical waveguide: the radiation of signal beam was focused onto the front wall of cell, while the radiation of the pump beam was focused at a distance of $2 \mathrm{~cm}$ from the front wall deep into a cell. It can be seen from Fig. $3 \mathrm{c}, \mathrm{d}$ that the dimensions of the signal beam gradually decreased at the output as the power of pump increased. Distinct changes in the distribution of signal beam radiation occurred at a pump intensity of $I=$ $20 \mathrm{MW} \mathrm{cm}^{-2}$. Raising intensity to $I=70 \mathrm{MW} \mathrm{cm}^{-2}$ allowed us to reduce the transverse dimensions of signal beam by two-three times (Fig. 3d). Upon raising the pump intensity further, the dimensions of signal beam began to increase slightly, as theoretical calculations predicted (Fig. 2). A more complicated transverse structure of the signal beam at the output from 
the cell (Fig. 3e) was observed by distortion of the signal beam focus far from the cell's front wall (in this case, the focuses of the pump and signal beams coincided). This could be due to the difraction effects on the deformed waveguide structure in the powerful pump beam's region of focusing.

\section{CONCLUSIONS}

Our theoretical analysis of interacting noncoherent laser beams in a medium with thermal nonlinearity allowed us to determine the conditions of attaining the mode of waveguide propagation of a signal light beam in the region of optical inhomogeneity induced by a powerful singular light beam. We experimentally demonstrated the localization of signal beam intensity near the waveguide axis upon collinear distribution of beams in an ethanol solution of $6 \mathrm{G}$ rhodamin.

\section{ACKNOWLEDGMENTS}

This work was supported by the Belarussian Republic Foundation for Basic Research (project no. F10R-070) and by the Russian Foundation for Basic Research (project no. 10-02-90010-Bel_a).

\section{REFERENCES}

1. Gibbs, H.M., Optical Bistability: Controlling Light by Light, Orlando: Acad. Press, 1985.

2. Kivshar Yu.S. and Agraval G.P., Optical Solitons: from Fibers to Photonic Crystals, San Diego: Academic Press, 2003; Moscow: Fizmatlit, 2005.

3. Dissipativnye solitony (Dissipative Solitons), Akhmediev, N. and Ankevich, A., Eds., Moscow: Fizmatlit, 2003.

4. Lobanov, V.E., Kalinovich, A.A., Sukhorukov, A.P., et al., Laser Phys., 2009, vol. 19, p. 1112.

5. Gorbach, D.V., Romanov, O.G., Sukhorukov, A.P., and Tolstik, A.L., Bull. Russ. Acad. Sci. Phys., 2010, vol. 74, no. 12 , p. 1637.

6. Kalinovich, A.A., Lobanov, V.E., Sukhorukov, A.P., and Tolstik, A.L., Bull. Russ. Acad. Sci. Phys., 2010, vol. 74, no. 12, p. 1718.

7. Dienerowitz, M., Mazilu, M., et al., Opt. Express, 2008, vol. 16, no. 7, p. 4991. 